\title{
Research Article \\ Detection of High-impact Movements in a Volleyball Match: A Cross-Sectional Study
}

\author{
Yasuharu Nagano $^{1 *}$ (D), Shogo Sasaki ${ }^{2}$ iD, Yuka Kose $^{1}$, Hiroshi Ichikawa ${ }^{3}$ \\ ${ }^{1}$ Department of Sports and Wellness Sciences, Japan Women's College of Physical Education, Tokyo, Japan \\ ${ }^{2}$ Faculty of Health Sciences, Tokyo Ariake University of Medical and Health Sciences, Tokyo, Japan \\ ${ }^{3}$ Department of Health and Sports, Niigata University of Health and Welfare, Niigata, Japan
}

\author{
Article Information \\ History: \\ Received: May 16, 2020 \\ Accepted: July 13, 2020 \\ Published: July 14, 2020

\section{Keywords:} \\ Athletic Injuries \\ Biomechanical Phenomena \\ Female \\ Prevention \& Control \\ Risk Assessment \\ Volleyball Injuries
}

\begin{abstract}
Objectives: Detection of the frequency or intensity of high-impact movements during volleyball match would help understand physical load causing the injuries. The present study aimed to classify different volleyball-specific high-impact movements based on the measurement of trunk acceleration in a volleyball match as a cross-sectional study.

Methods: Linear accelerations of six female volleyball players were measured during a single volleyball match. The instances at which $>4 \mathrm{G}$ and $>6 \mathrm{G}$ of resultant acceleration was generated were detected. Movements recorded with a synchronized video were also detected and categorized. Counts (cases) and frequencies (cases/min) of the detected movements were calculated, and the resultant and each directional acceleration among the top seven volleyball-specific detected movements were compared.

Results: For attackers, 361 and 97 movements were detected for the resultant acceleration thresholds over $4 \mathrm{G}$ and $6 \mathrm{G}$, respectively. Landing, takeoff and running were the top three detected movements in both thresholds. Landing was the most frequently observed high-impact movement, and the ratio of landing over $6 \mathrm{G}$ was greater among all detected movements compared with the ratios of landing over 4G threshold. For receivers, 297 and 38 movements were detected for the resultant acceleration thresholds over $4 \mathrm{G}$ and $6 \mathrm{G}$, respectively. Running, steps on the spot, and steps forward or backward were the top three detected movements. The top seven detected volleyball-specific movements generating over 4G were landing after blocking, landing after spiking, takeoff before spiking, takeoff before blocking, stationary steps during receiving, steps forward or backward during receiving and sidesteps during receiving. Resultant and vertical accelerations of landing after spiking were significantly greater than those of the other six movements $(p<0.001)$.
\end{abstract}

Conclusions: Using the results of present study, we were able to estimate the physical load causing injuries during a real volleyball match.

\section{INTRODUCTION}

$\mathrm{P}$ hysical load during sports activities has been evaluated based on training time or match exposure [1], rate of perceived exertion $[2,3]$, heart rate $[2,4]$ or speeds and distance from tracking data [5]. However, these indices are inadequate for the quantification of muscular skeletal load or risk of injury. The risk of musculoskeletal injury has been frequently assessed thru evaluation of movements using a

*Correspondence: Yasuharu Nagano, PhD. 8-19-1 Kitakarasuyama, Setagaya Tokyo 157-8565, Japan. Tel: +81-3-3300-5437; Fax: +81-3-3300-5437

E-mail address: nagano.yasuharu@jwcpe.ac.jp motion capture system comprised of reflective markers and a force plate. Indeed, it has been reported that screening of landing movements under laboratory conditions cannot predict the risk of injury [6]. Therefore, the evaluation of movement during sports activities for effective understanding of movement or for physical load is required.

Volleyball is a sport that typically involves high-impact movements. Knee injuries are common in volleyball, which is among the top three most common injuries as with ankle and shoulder injuries [7]. Knee injuries in volleyball occur both as acute and as overuse [7]. High-impact movements in volleyball often cause injuries such as anterior cruciate ligament (ACL) rupture [8] and jumper's knee [1]. Previous studies $[1,8]$ suggested that high-impact landing during volterms of the Creative Commons Attribution License (http://creativecommons.org/licenses/by/4.0/), which permits unrestricted use, distribution, and reproduction in any medium, provided the original work is properly cited. 
leyball practice and matches can result in acute and overuse injuries. Therefore, detection of the frequency or intensity of high-impact movements would help understand physical load causing the injuries.

Recently, lightweight and wearable inertia sensors have been used for the assessment of movement. Simons and Bradshaw reported that the impact load during jumping and landing tasks and GRF could be reliably assessed using accelerometry $[9,10]$. Moreover, vertical acceleration during change of direction can potentially reflect gender differences and stiff cutting movement [11]. Inertia sensors demonstrating excellent accuracy counting volleyball-specific jumps is an example of its application during sports activity [12-14]. Therefore, using these methods, high-impact movements and evaluation of these movements can potentially be evaluated to define physical load during sports activity. However, these previous studies were conducted in a laboratory setting [9-11] or did not show the details of the high-impact movement increasing the physical load during the volleyball match [12-14].

The aim of the present study was to classify the details of volleyball-specific high-impact movements based on the measurement of trunk acceleration during a volleyball match. Comparison of acceleration values between volleyball-specific movements is another purpose of this study. We hypothesized that spike landings would be associated with higher trunk accelerations compared with other movements. Additionally, we hypothesized that spike landing would be associated with a higher vertical acceleration compared with other movements, thereby reflecting movement characteristics.

\section{METHODS}

\section{Participants}

In total, six female volleyball players (age, $20.0 \pm 0.6$ years; body mass, $59.3 \pm 7.1 \mathrm{~kg}$; height, $160.7 \pm 7.3 \mathrm{~cm}$; years of experience, $10.7 \pm 2.9$ years [mean \pm SD] ) were enrolled for the experiment. The participants played a nine-person volleyball match with fixed player positions. The six participants conducted a measurement as a same team. Three of the participants were attackers, who were mainly active in the front row of the court, whereas the remaining were receivers, who were mainly active at the back row of the court. The exclusion criteria after physical evaluation were history of serious musculoskeletal injury, any musculoskeletal injury within the past 3 months, or any disorder that interfered with sensory input, musculoskeletal function, or motor function. Before study participation, all participants and their parents provided written, informed consent according to the requirements of the ethical review board of authors' affiliation.

\section{Measurements}

A Linear acceleration was measured using a lightweight, triaxial accelerometer with a full-scale range of \pm 16 G (SS-
WS1201, Sports Sensing, Fukuoka, Japan). The accelerometer was secured to the upper back of the participant using an exclusive vest with compressive inner wear that did not prevent their upper or lower limb movements [15]. We selected trunk acceleration as a variable indicating musculoskeletal load because it more reliably assesses GRF than knee acceleration does [11], and it does not prevent the upper and lower limb movements of athletes during measurement. The three axes of the accelerometer were aligned close to the anatomical axes i.e. the $\mathrm{X}$-axis was aligned mediolaterally, the Y-axis vertically and the Z-axis anteroposteriorly. Acceleration data were sampled at a frequency of $200 \mathrm{~Hz}$ and saved in the built-in memory of the accelerometer. The axial direction of the accelerometer was set at $+1 G$, indicating acceleration due to gravity.

Using video recordings $(60 \mathrm{~Hz})$ from a digital video camera (GZ-RX600, JVC, Japan), we measured the linear acceleration during a single volleyball match consisting of 21 total points versus a team at the same competition level. The cameras were positioned approximately $20 \mathrm{~m}$ from the center of the end line at the player level (a height of approximately $1.3 \mathrm{~m}$ ) to capture players' movements from the back. We recorded the movements of the players throughout the entire match without panning or tilting. All videos were stored in high-definition (1080i) AVCHD format. A light pulse was used at the start of the measurement to synchronize the accelerometer with the video sequence from the start of the timing.

The total time of the match was 18 minutes and $25 \mathrm{sec}-$ onds including the time when the play was stop between points. The final score was 21-9. After testing, the accelerometer was connected to a computer, and raw data were downloaded into a database for later analyses. In our pilot study (unpublished), to confirm the reliability of determining the frequency of detected movement, we calculated the intraclass correlation coefficient (ICC) $(1,1)$ of the number of extracted movements at which $>4 \mathrm{G}$ of resultant acceleration was generated for 11 volleyball players among three sets of volleyball match. The resulting ICC was 0.80 indicating high reliability.

The resultant acceleration was calculated from the acceleration data. A customized tagging application (Sports Sensing, Fukuoka, Japan) was used to detect instances at which $>4 \mathrm{G}$ and $>6 \mathrm{G}$ of resultant acceleration was generated during a match. Because a previous study [15] showed that thresholds greater than $4 \mathrm{G}$ could adequately detect the landing during badminton games, we adopted this threshold for the current research. In addition, a threshold greater than $6 \mathrm{G}$ was temporarily used to examine the ratio of extracted movements by each threshold, especially in volleyball. By using the Dartfish software (Connect Plus, Dartfish, Switzerland), video sequences at the instances they were generated at $>4 \mathrm{G}$ and $>6 \mathrm{G}$ resultant accelerations were detected and made to agree with the time of extracted acceleration data. We categorized the movements as landing, takeoff, running, swing, sidesteps, stop, cutting, steps forward or backward, stationary steps, sliding and other movements based on de- 
tected video image. Furthermore, we categorized the details of volleyball-specific movements as spiking, blocking, receiving and positioning. Categorization was performed by two researchers who were required to reach a unanimous decision; when disagreement occurred, other researchers were consulted to obtain a consensus and final decision.

\section{Statistical Analysis}

For each acceleration threshold ( $>4 \mathrm{G}$ and $>6 \mathrm{G}$ ), counts (cases) and frequencies (cases/min) of the detected movements in each position were calculated. The $95 \%$ confidence interval (CI) was calculated for the frequencies of all detected movements and the top three frequent movements of each subjects using the following formula: movement rate \pm 1.96 $\times$ (movement rate/square root [number of movements]) [16]. To assess the number of cases required to determine significant differences between the resultant accelerations observed in the seven groups of volleyball-specific movements, statistical power analysis was performed to achieve $80 \%$ statistical power with an a level of 0.05 and an effects size of 0.4 . The analysis showed that at least 20 cases in total were required to achieve sufficient statistical power. Therefore, we extracted the top seven movements that generated a resultant acceleration of $>4 \mathrm{G}$ among the combination of volleyball-specific movements. We compared the resultant and each directional acceleration (mediolateral, vertical and anteroposterior) of the top seven extracted movements using a two-way analysis of variance (ANOVA) (movements, subjects) test to examine main effect and interaction, along with Bonferroni correction performed as a post hoc test. In vertical acceleration, a plus value indicated an upward direction, whereas in anteroposterior acceleration, a plus value indicated a posterior direction. For mediolateral acceleration, absolute values were used to eliminate the influence of position of the dominant hand. For all experiments, $\mathrm{p}<0.05$ was considered statistically significant.

\section{RESULTS}

For attackers, the counts and frequency at each resultant acceleration threshold $(>4 \mathrm{G}$ and $>6 \mathrm{G}$ ) for each detected movement is detailed in Table 1. A total of 361 and 97 movements were detected for the resultant acceleration thresholds over $4 \mathrm{G}$ and $6 \mathrm{G}$, respectively. Landing, takeoff and running were the top three detected movements in both thresholds. The ratio of landing over $6 \mathrm{G}$ was greater among all detected movements compared with the ratios of landing over $4 \mathrm{G}$ threshold. For receivers, the counts and frequency at each resultant acceleration threshold $(>4 \mathrm{G}$ and $>6 \mathrm{G}$ ) for each detected movement is detailed in Table 2. A total of 297 and 38 movements were detected for the resultant acceleration thresholds over $4 \mathrm{G}$ and $6 \mathrm{G}$, respectively. Running, steps on the spot and steps forward or backward were the top three detected movements; however, steps on the spot and steps forward or backward were hardly detected using over $6 \mathrm{G}$ threshold.

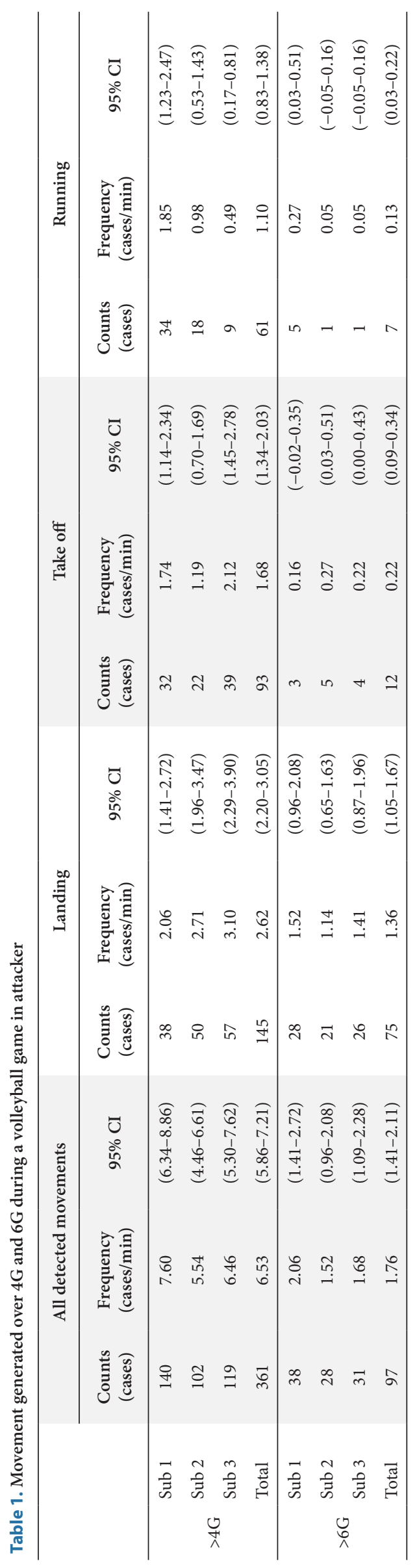

Exercise Medicine | 3 


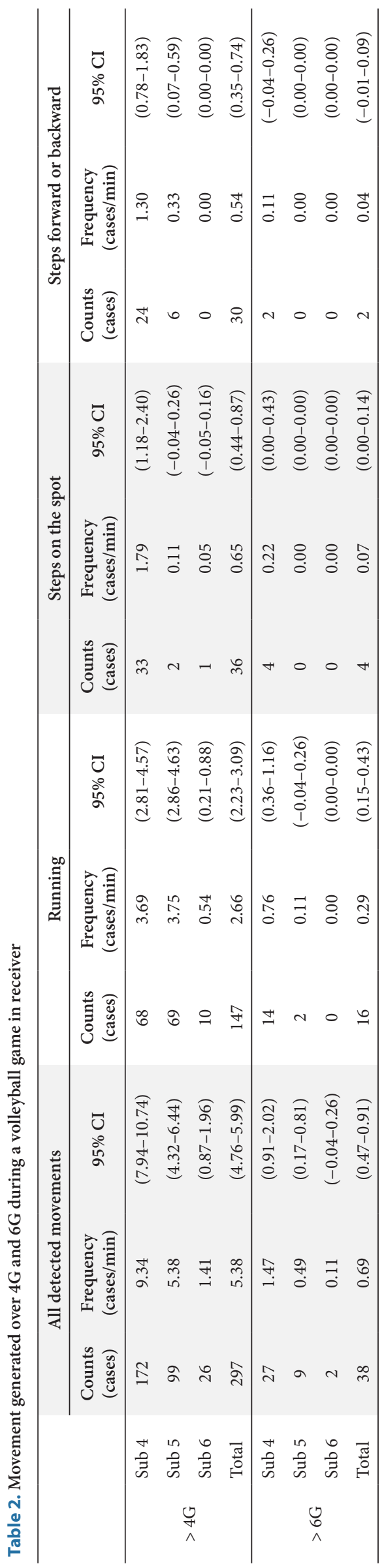

The top seven detected volleyball-specific movements generating over $4 \mathrm{G}$ were landing after blocking, landing after spiking, takeoff before spiking, takeoff before blocking, stationary steps during receiving, steps forward or backward during receiving and sidesteps during receiving. Movement and subject as factors in the resultant, mediolateral, vertical and anteroposterior accelerations are shown in Table 3 using a two-way ANOVA summary. The descriptive statistics of the resultant, mediolateral, vertical and anteroposterior accelerations for the top seven extracted movements are presented in Table 4.

\section{DISCUSSION}

The present study revealed the movements that generate the high impact based on trunk accelerations and these frequencies during a volleyball match. Our findings demonstrate that in attackers, landing was the most frequently observed high-impact movement, and the ratio of landing movements increased the most with increasing acceleration threshold among all detected high loaded movements. In receivers, running was the most frequently observed movement; however, the number of detected movements decreased with increasing threshold. In terms of the magnitude associated with high loaded movements, landing after spiking showed the greatest resultant and vertical acceleration among all the other movements, and stationary and directional steps showed the greatest mediolateral and anteroposterior acceleration among all the other movements. High loaded movements measured by greater acceleration means high-impact and poor buffering posture at the time of movements. In a recent study, acceleration was measured by torso-mounted inertial sensors that reported normal variables indicating the external demands during running and the changes of direction [17] or sport-specific movements [15,18]. Using these findings, we were able to estimate the physical load.

Landing was identified as the most frequently observed movement with the greatest magnitude during a volleyball match. In the case of volleyball, the landing position following a spike is more upright than other volleyball movements because it occurs near the net. Landing in the upright posture increases the GRF [19], and acceleration during landing with decreased trunk inclination and knee flexion angle is thought to be greater. The results reported that the impact during landing after spiking was greater than after blocking, which agreed to a previous study [20]. Unfortunately, landing after spiking has been investigated in only a limited number of studies, whereas the risk of ACL injury during landing after blocking has been assessed in several previous studies [21-23]. Landing after spiking should be examined as a higher-risk movement that could result in ACL injury during volleyball matches. Moreover, the risk of ACL injury in athletes who demonstrate frequent or high-impact landing during volleyball matches should be examined in future studies.

Jumper's knee is one of the overuse injuries caused by 
Table 3. Two-way ANOVA summary for movement and subject as factors in each acceleration

\begin{tabular}{|c|c|c|c|c|c|c|c|}
\hline Variables & Factor & $\mathrm{df}$ & SS & MS & $\eta 2$ & F value & $\mathrm{p}$ values \\
\hline \multirow[t]{3}{*}{ Resultant } & Movement (M) & 6 & 190 & 31.72 & 0.22 & 13.6 & $<0.001$ \\
\hline & Subject (S) & 5 & 31 & 6.13 & 0.04 & 2.6 & $<0.05$ \\
\hline & $M * S$ & 12 & 141 & 11.77 & 0.17 & 5.0 & $<0.001$ \\
\hline \multirow[t]{3}{*}{ Mediolateral } & Movement (M) & 6 & 16 & 2.66 & 0.12 & 6.9 & $<0.001$ \\
\hline & Subject (S) & 5 & 7 & 1.45 & 0.06 & 3.8 & $<0.01$ \\
\hline & $M * S$ & 12 & 12 & 0.98 & 0.09 & 2.5 & $<0.01$ \\
\hline \multirow[t]{3}{*}{ Vertical } & Movement (M) & 6 & 158 & 26.25 & 0.16 & 8.9 & $<0.001$ \\
\hline & Subject (S) & 5 & 39 & 7.83 & 0.04 & 2.7 & $<0.05$ \\
\hline & $M * S$ & 12 & 149 & 12.42 & 0.15 & 4.2 & $<0.001$ \\
\hline \multirow[t]{3}{*}{ Anteroposterior } & Movement (M) & 6 & 16 & 2.68 & 0.05 & 2.5 & $<0.05$ \\
\hline & Subject (S) & 5 & 2 & 0.42 & 0.01 & 0.4 & N.S. \\
\hline & $M * S$ & 12 & 38 & 3.20 & 0.11 & 2.9 & $<0.001$ \\
\hline
\end{tabular}

Table 4. Frequency and components of each acceleration (mean (SD)) among top 7 frequent movements generating over $4 \mathrm{G}$

\begin{tabular}{|c|c|c|c|c|c|c|c|c|c|c|c|}
\hline \multirow{2}{*}{$\begin{array}{c}\text { Movement } \\
\text { Landing after blocking }\end{array}$} & \multirow{2}{*}{$\begin{array}{c}\begin{array}{c}\text { Counts } \\
\text { (cases) }\end{array} \\
76\end{array}$} & \multirow{2}{*}{$\begin{array}{r}\begin{array}{r}\text { Frequency } \\
\text { (cases/min) }\end{array} \\
1.38\end{array}$} & \multirow{2}{*}{$\begin{array}{l}95 \% \mathrm{CI} \\
(1.07-1.69)\end{array}$} & \multicolumn{2}{|c|}{ Resultant (G) } & \multicolumn{2}{|c|}{$\begin{array}{c}\text { Mediolateral } \\
(\mathrm{G})\end{array}$} & \multicolumn{2}{|c|}{ Vertical (G) } & \multicolumn{2}{|c|}{ Anteroposterior (G) } \\
\hline & & & & 5.91 & $(1.41)^{\mathrm{a}}$ & 0.90 & $(0.61)$ & 5.51 & $(1.77)^{\mathrm{e}}$ & -0.12 & $(1.52)$ \\
\hline Landing after spike & 62 & 1.12 & $(0.84-1.40)$ & 7.76 & $(3.13)^{\mathrm{b}}$ & 1.09 & $(0.93)$ & 7.41 & $(3.37)^{\mathrm{b}}$ & 0.33 & $(1.29)$ \\
\hline Takeoff before spike & 48 & 0.87 & $(0.62-1.12)$ & 5.30 & $(1.07)$ & 0.70 & $(0.47)$ & 5.20 & $(1.07)$ & 0.22 & $(0.55)$ \\
\hline Takeoff before blocking & 40 & 0.72 & $(0.50-0.95)$ & 4.83 & $(0.89)$ & 0.41 & $(0.39)$ & 4.64 & $(0.95)$ & 0.31 & $(1.13)$ \\
\hline $\begin{array}{l}\text { Steps on the spot during } \\
\text { receive }\end{array}$ & 36 & 0.65 & $(0.44-0.87)$ & 5.01 & $(0.96)$ & 1.12 & $(0.69)^{c}$ & 4.61 & (1.08) & 1.24 & $(0.59)^{\mathrm{f}}$ \\
\hline $\begin{array}{l}\text { Steps forward or backward } \\
\text { during receive }\end{array}$ & 30 & 0.54 & $(0.35-0.74)$ & 4.74 & $(0.92)$ & 1.27 & $(0.66)^{d}$ & 4.29 & $(1.02)$ & 1.25 & $(0.51)^{\mathrm{g}}$ \\
\hline Sidesteps during receive & 23 & 0.42 & $(0.25-0.59)$ & 4.99 & $(0.85)$ & 1.32 & $(0.65)^{\mathrm{d}}$ & 4.56 & $(0.92)$ & 1.22 & $(0.68)^{\mathrm{h}}$ \\
\hline
\end{tabular}

'Significantly greater than 'takeoff before blocking' and 'steps forward or backward during receive' $(p<0.01)$

${ }^{b}$ Significantly greater than other six movements $(p<0.001)$

'Significantly greater than 'takeoff before blocking' $(p<0.001)$

${ }^{d}$ Significantly greater than 'takeoff before spike' and 'takeoff before blocking' ( $p<0.01$ and $p<0.001$, respectively)

'Significantly greater than 'steps forward or backward during receive' $(p<0.05)$

'Significantly greater than 'landing after blocking', 'landing after spike', 'takeoff before spike', and 'takeoff before blocking' ( $p<0.001, p<0.01, p<0.001$, and $p<0.01$, respectively)

sSignificantly greater than 'landing after blocking', 'landing after spike', 'takeoff before spike', and 'takeoff before blocking' ( $p<0.001, p<0.01, p<0.01$, and $p<0.01$, respectively

'Significantly greater than 'landing after blocking', 'landing after spike', 'takeoff before spike', and' 'takeoff before blocking' ( $p<0.001, p<0.05, p<0.01$, and $p<0.05$, respectively)

repeated load, which particularly occurs during landing [1]. In another study [12-14] where a different algorithm was used, using an inertia sensor could help increase the precision in determining the load of landing because the acceleration was reported as an applicable method for estimating jump counts in volleyball. Although the present study demonstrated interindividual differences that are also observed in a previous study [20], we observed that almost all movements detected at an acceleration above the 6G threshold coincided with jump-landing movements in the attacker. Therefore, if we set appropriate threshold for each subject group, the frequency of movements with accelerations may be used as an index of jump-landing load during volleyball.

The following are some limitations of the present study.
First, we measured movements only in a single volleyball match with a small number of participants. It is possible that fatigue or change of surroundings could affect acceleration. For example, in previous studies, the peak vertical ground reaction force during landing was increased in the fatigue condition with decreased trunk inclination and knee range of motion [24] as well as less cushioned shoes [25]. Additionally, as the number of participants increases, an increase in detailed categorization is possible. However, the present study provides basic data on movements during a single volleyball match. In future studies, the effect(s) of the number of matches, details of matches, or the material of shoes and the floor on acceleration should be investigated in a large population study. Second, the present study was a cross-sectional study. To prove the relationship between 
trunk acceleration and injury development, a prospective cohort study is necessary. It is particularly important to determine which index and threshold are associated with injuries.

\section{CONCLUSIONS}

The present study classified different volleyball-specific high-impact movements based on the measurement of trunk acceleration in a volleyball match. Landing was the most frequently observed high-impact movement, and the ratio of landing movements increased the most with increasing acceleration threshold among all detected high loaded movements. Landing after spiking have the greatest magnitude during the volleyball match. Using the results of present study, we were able to estimate the physical load during a real volleyball match.

\section{ACKNOWLEDGEMENTS}

The authors would like to thank Enago for the English language review.

\section{Conflicts of Interest}

The authors declare no conflict of interest.

\section{Funding}

This work was supported by a JSPS KAKENHI Grant-inAid for Young Scientists (B) (Grant Number 26870669).

\section{REFERENCES}

1. Visnes H, Bahr R. Training volume and body composition as risk factors for developing jumper's knee among young elite volleyball players. Scand J Med Sci Sports. 2013; 23(5):607-613.

2. Casamichana D, Castellano J, Calleja-Gonzalez J, San Roman J, Castagna C. Relationship between indicators of training load in soccer players. J Strength Cond Res. 2013; 27(2):369-374.

3. Hulin BT, Gabbett TJ, Blanch P, Chapman P, Bailey D, Orchard JW. Spikes in acute workload are associated with increased injury risk in elite cricket fast bowlers. Br J Sports Med. 2014; 48(8):708-712.

4. Scribbans TD, Berg K, Narazaki K, Janssen I, Gurd BJ. Heart rate during basketball game play and volleyball drills accurately predicts oxygen uptake and energy expenditure. J Sports Med Phys Fitness. 2015; 55(9):905913.

5. Malone JJ, Lovell R, Varley MC, Coutts AJ. Unpacking the Black Box: Applications and Considerations for Using GPS Devices in Sport. Int J Sports Physiol Perform. 2017; 12(Suppl 2):S218-S226.

6. Krosshaug T, Steffen K, Kristianslund E, et al. The Ver- tical Drop Jump Is a Poor Screening Test for ACL Injuries in Female Elite Soccer and Handball Players: A Prospective Cohort Study of 710 Athletes. Am J Sports Med. 2016; 44(4):874-883.

7. Kilic O, Maas M, Verhagen E, Zwerver J, Gouttebarge V. Incidence, aetiology and prevention of musculoskeletal injuries in volleyball: A systematic review of the literature. Eur J Sport Sci. 2017; 17(6):765-793.

8. Devetag F, Mazzilli M, Benis R, A LAT, Bonato M. Anterior cruciate ligament injury profile in Italian Serie A1-A2 women's volleyball league. J Sports Med Phys Fitness. 2018; 58(1-2):92-97.

9. Simons C, Bradshaw EJ. Reliability of accelerometry to assess impact loads of jumping and landing tasks. Sports Biomech. 2016; 15(1):1-10.

10. Simons C, Bradshaw EJ. Do accelerometers mounted on the back provide a good estimate of impact loads in jumping and landing tasks? Sports Biomech. 2016; 15(1):76-88.

11. Nagano Y, Sasaki S, Higashihara A, Ishii H. Gender differences in trunk acceleration and related posture during shuttle run cutting. Int Biomech. 2016; 3(1):33-39.

12. Charlton PC, Kenneally-Dabrowski C, Sheppard J, Spratford W. A simple method for quantifying jump loads in volleyball athletes. J Sci Med Sport. 2017; 20(3):241-245.

13. MacDonald K, Bahr R, Baltich J, Whittaker JL, Meeuwisse WH. Validation of an inertial measurement unit for the measurement of jump count and height. Phys Ther Sport. 2017; 25:15-19.

14. Skazalski C, Whiteley R, Hansen C, Bahr R. A valid and reliable method to measure jump-specific training and competition load in elite volleyball players. Scand J Med Sci Sports. 2018; 28(5):1578-1585.

15. Nagano Y, Sasaki S, Higashihara A, Ichikawa H. Movements with greater trunk accelerations and their properties during badminton games. Sports Biomech. 2018:1-11.

16. Tscholl P, O'Riordan D, Fuller CW, Dvorak J, Junge A. Tackle mechanisms and match characteristics in women's elite football tournaments. Br J Sports Med. 2007; 41 Suppl 1:i15-19.

17. Wundersitz DW, Netto KJ, Aisbett B, Gastin PB. Validity of an upper-body-mounted accelerometer to measure peak vertical and resultant force during running and change-of-direction tasks. Sports Biomech. 2013; 12(4):403-412.

18. Staunton C, Wundersitz D, Gordon B, Kingsley M. Construct Validity of Accelerometry-Derived Force to Quantify Basketball Movement Patterns. Int J Sports Med. 2017; 38(14):1090-1096.

19. Shimokochi Y, Ambegaonkar JP, Meyer EG. Changing Sagittal-Plane Landing Styles to Modulate Impact and Tibiofemoral Force Magnitude and Directions Relative to the Tibia. J Athl Train. 2016; 51(9):669-681.

20. Jarning JM, Mok KM, Hansen BH, Bahr R. Application of a tri-axial accelerometer to estimate jump frequency 
in volleyball. Sports Biomech. 2015; 14(1):95-105.

21. Favre J, Clancy C, Dowling AV, Andriacchi TP. Modification of Knee Flexion Angle Has Patient-Specific Effects on Anterior Cruciate Ligament Injury Risk Factors During Jump Landing. Am J Sports Med. 2016; 44(6):1540-1546.

22. Zahradnik D, Jandacka D, Farana R, Uchytil J, Hamill J. Identification of types of landings after blocking in volleyball associated with risk of ACL injury. Eur J Sport Sci. 2017; 17(2):241-248.

23. Zahradnik D, Jandacka D, Holcapek M, Farana R,
Uchytil J, Hamill J. Blocking landing techniques in volleyball and the possible association with anterior cruciate ligament injury. J Sports Sci. 2018; 36(8):955-961.

24. Wong TL, Huang CF, Chen PC. Effects of Lower Extremity Muscle Fatigue on Knee Loading During a Forward Drop Jump to a Vertical Jump in Female Athletes. J Hum Kinet. 2020; 72:5-13.

25. Fu W, Fang Y, Gu Y, Huang L, Li L, Liu Y. Shoe cushioning reduces impact and muscle activation during landings from unexpected, but not self-initiated, drops. J Sci Med Sport. 2017; 20(10):915-920. 